\title{
Mathematical modeling highlights from ARVO 2018
}

Kara L. Maki ${ }^{1}$, Rodolfo Repetto², Richard J. Braun ${ }^{3}$

${ }^{1}$ School of Mathematical Sciences, Rochester Institute of Technology, Rochester, NY, USA; ${ }^{2}$ Department of Civil, Chemical and Environmental Engineering, University of Genoa, Genoa, Italy; ${ }^{3}$ Department of Mathematical Sciences, University of Delaware, Newark, DE, USA

Keywords: anterior chamber, dry eye, modeling, posterior chamber, retina, tear film

At the ARVO annual meeting, there is an increasing number of contributions that involve significant mathematical modeling of ocular physiology and procedures. There has long been significant use of statistical methods for understanding data from a variety of in vivo measurements and clinical trials. Beyond these important uses of statistical and mathematical tools, a growing number of researchers are developing mathematical and computational models, often based on fundamental principles from physics, chemistry and mechanics, that provide insights into ocular phenomena. A number of areas had noticeable contributions involving applications of models, such as tear production, tear film dynamics, corneal biomechanics, retinal blood flow, and glaucoma. We list a number of such contributions in this introduction and follow those with five extended abstracts that summarize some of the studies mentioned here.

Guidoboni et al. ${ }^{1}$ studied fluid flow in the eye and related organs by using a lumped parameter model. Their model accounts for the flows of blood and aqueous humor in the eyes and the flows of blood, cerebrospinal fluid, and interstitial fluid in the brain, as well as their interactions. Eye and brain are connected to a simple model of the whole-body circulation. The model captures the major observed trends in the interactions between cerebrospinal fluid pressure, intraocular pressure (IOP), and choroidal venule pressure.

Ishihara ${ }^{2}$ used a mathematical model to simulate transmission from cones to OFF type cone bipolar cells (OFF-BCs) and the role of ionic currents in this process. In the

Correspondence: Richard J. Braun, Department of Mathematical Sciences, University of Delaware, Newark, DE 19716, USA. E-mail: rjbraun@udel.edu 
cone cells, the model focused on the role of glutamate and its receptors, as well as calcium ions, after the cells are stimulated by a photosensitive current model. In the OFF-BCs, the model focused on rectifying currents that may involve potassium ions. The two cells were coupled together in the model, and parameters were determined from mammalian retinas where possible. Frequency characteristics of the system were found, suggesting a band-pass behavior. At lower light levels, the OFF-BC response was suppressed; however, transient signals could still be sent at low light levels.

Maki et al. ${ }^{3}$ studied the influence of the blink on the formation of a stable tear film. They developed a numerical method to approximate a simplified mathematical model characterizing the tear film dynamics during a realistic blink. The model captures the flow of the aqueous tears on the exposed ocular surface, and accounts for influxes and effluxes of aqueous tears at the lid margin during the blink. It was found that sufficient aqueous tears must be supplied along the lid margin near the exposed ocular surface and must be supplied at rates comparable to the speed of ocular exposure to ensure the formation of a stable tear film with "healthy" menisci. These simulations will allow for the continued exploration of the tear dynamics in regions on the ocular surface not accessible to imaging techniques due to interference by lid motion.

Yabusaki et $a .^{4}$ are the first group, to our knowledge, to publish on machine learning $(\mathrm{ML})$ techniques applied to tear film imaging and dry eye diagnosis. Using interference fringe patterns and colors form the tear film lipid layer (TFLL) as input, they aimed to diagnose differences between aqueous deficient dry eye (ADDE), evaporative dry eye (EDE), and normals. A linear support vector machine-based $M L$ approach was compared to diagnoses by one of the co-authors (who is experienced at diagnosis). Better agreement was obtained for ADDE than for EDE or normal-type predictions. This approach opens the door to an exciting path of investigation for ocular surface researchers and clinicians.

Cwiklik et al. ${ }^{5}$ simulated the dynamics of the TFLL in the presence of the preservative benzalkonium chloride (BAK) with a few different hydrocarbon chain lengths. These short-chain molecules are surface active and similar to cetalkonium chloride (CKC). The latter was previously shown to affect the TFLL, improving its stability. Their detailed simulations showed that the BAK molecules, particularly the shortest one, could orient itself through a wide range of angles relative to the TFLL/aqueous interface. Longer molecules like CKC could not do this. These results help explain the destabilizing effect of short-chain BAK molecules.

Zhong et al. ${ }^{6}$ study the tear breakup (TBU) caused by local "globs" of excess lipid. The surface active globs cause rapid divergent (outward) flow that can lead to rapid TBU. They also included evaporation of the aqueous to surrounding air, osmotic supply of aqueous from the epithelium, and transport of solutes such as fluorescein (FL). From computed tear film thickness and FL concentration, they could compute 
the emitted FL intensity. They then computed what fluorescent images would look like in experiments and determined desirable initial concentration ranges for imaging evaporative (slow) vs rapid (divergent flow) instances of TBU.

There were also math-focused presentations in the areas of corneal biomechanics, glaucoma, and more. For example, Tavakol et al. ${ }^{7}$ simulated the response of the cornea to mechanical stimuli. The long-term goal is to estimate corneal elasticity by measuring the speed of acoustic wave propagation. The authors presented a finite element (FE) model created in COMSOL, where they probed how individual parameters affected corneal wave propagation.

Missel and Sarangapani ${ }^{8}$ presented a computational multi-physics model to study the potential benefit of implanting more than one supraciliary micro-stent in cataract surgery. The micro-stent is implanted to lower IOP in glaucoma patients. Their modeling efforts found a single micro-stent is sufficient in equilibrating the IOP with the suprachoroidal pressure.

Jin et al. ${ }^{9}$ used FE analysis to model the origin of the ocular pulse and to investigate the role of biomechanical factors on optic nerve head $(\mathrm{ONH})$ deformations during the cardiac cycle. The authors constructed a FE model of the eye and modelled the choroid as a biphasic material, constituted of a solid phase (connective tissues) and a fluid phase (blood). The model shows that fluctuations of the arterial blood pressure produce significant IOP changes and ONH deformations. Systolic ophthalmic artery pressure and choroidal stiffness are found to be the parameters with the strongest influence.

Chuangsuwanich et $a l^{10}$ proposed a computational model to study oxygen concentration and consumption in the retinal ganglion cell axons in the lamina cribrosa (LC). They generated many artificial LC micro-capillary networks, in which they simulated numerically blood flow and oxygen diffusion and consumption. The model highlights the heterogeneous distribution of oxygen concentration within the LC, which may contribute to spatial variability of axonal loss in glaucoma.

We hope that you enjoy the extended abstracts here as much as we enjoyed learning about these and other results from ARVO 2018.

\section{Acknowledgements}

Richard J. Braun was supported by National Science Foundation (NSF) grant DMS 1412085. Kara Lee Maki was supported by NSF grant DMS 1412141. 


\section{References}

1. Guidoboni G, Salerni F, Repetto R, Szopos M, Harris A. Relationship between intraocular pressure, blood pressure and cerebrospinal fluid pressure: a theoretical approach. Invest Ophthalmol Vis Sci. 2018;59(9):1665.

2. Ishihara A. A simulation study of information transmission in OFF cone bipolar cell pathway. Invest Ophthalmol Vis Sci. 2018;59(9):1873.

3. Maki K, McManus A, Henshaw W, Braun R. The influence of tear supply and drainage on tear film dynamics during a realistic blink. Invest Ophthalmol Vis Sci. 2018;59(9):3817.

4. Yabusaki K, Arita R, Yamauchi T. Automated classification of dry eye type analyzing interfering fringe color images of tear film using machine learning technologies. Invest Ophthalmol Vis Sci. 2018;59(9):4860.

5. Cwiklik L, Riedlova K, Melcrova A, Olzynska A, Daull P, Garrigue J. Influence of benzylalkonium chloride on tear film lipid layer stability: a molecular level view by employing in silico modeling. Invest Ophthalmol Vis Sci. 2018;59(9):3279.

6. Zhong L, Antwi D, Braun R, King-Smith P, Begley C. Simulation and measurement of glob-driven tear film breakup. Invest Ophthalmol Vis Sci. 2018;59(9):4907.

7. Tavakol B, Birkenfeld J, Ramier A, Yun S-H. Development of a time-domain finite-element model of acoustic wave propagation in the cornea. Invest Ophthalmol Vis Sci. 2018;59(9):1401.

8. Missel P, Sarangapani R.Comparing the predicted efficacy of one versus two supraciliary micro-stents for lowering IOP in glaucoma patients using an ocular computational fluid dynamic model. Invest Ophthalmol Vis Sci. 2018;59(9):2057.

9. Jin Y,Wang X, Zhang L, Jonas J, Aung T, Schmetterer L, et al. Finite element modeling predicts neural tissue shear in the neuroretinal rim caused by pulsatile blood pressure. Invest Ophthalmol Vis Sci. 2018;59(9):2037.

10. Chuangsuwanich T, Leo L, Schmetterer L, Girard MJ. An hourglass pattern of oxygen distribution in the lamina cribrosa predicted using a computational model. Invest Ophthalmol Vis Sci. 2018;59(9):4477. 